NISSUNA UMANA INVESTIGAZIONE SI PUO DIMANDARE VERA SCIENZIA S'ESSA NON PASSA PER LE MATEMATICHE DIMOSTRAZIONI LEONARDO DA VINCI

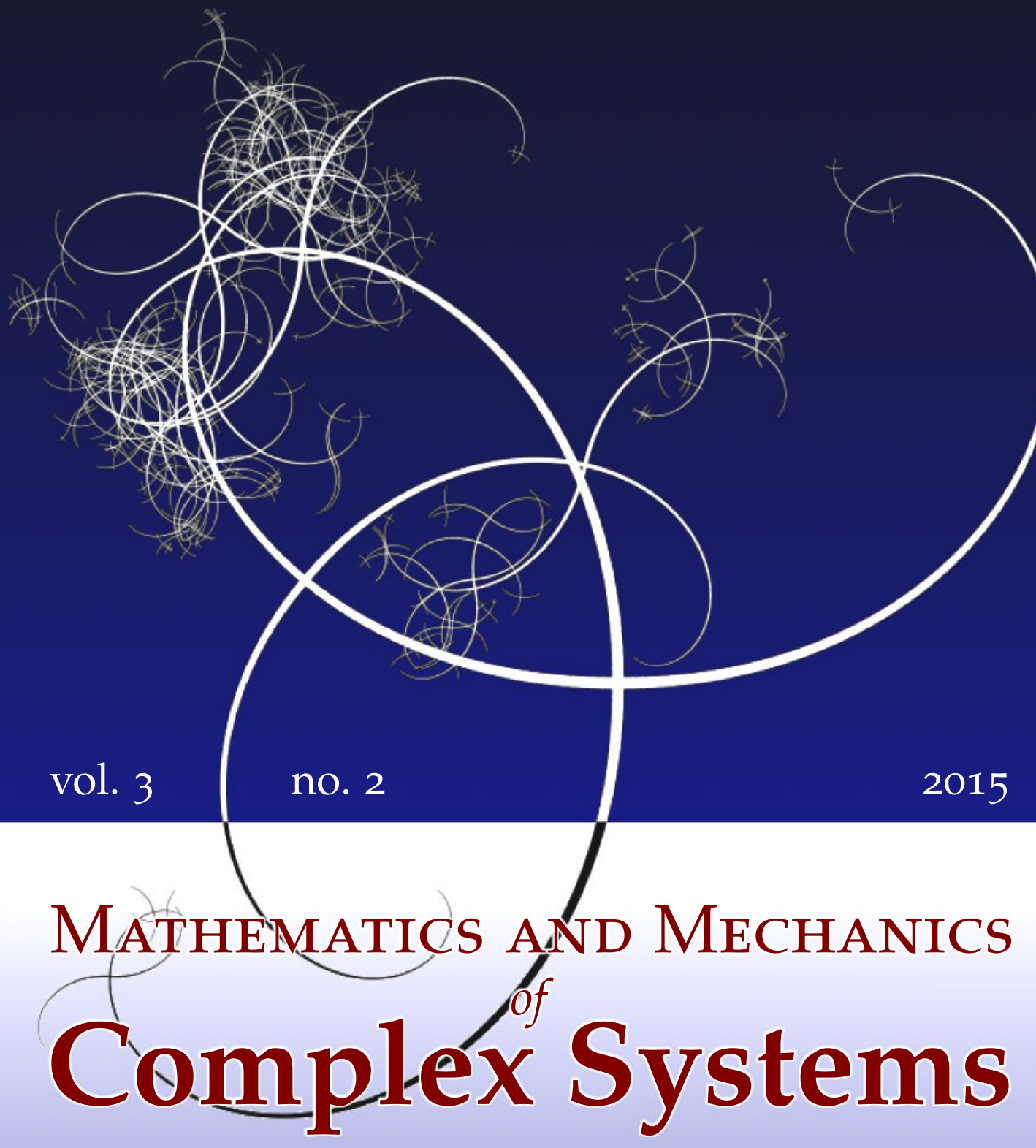

Xu Wang And Peter Schiavone

NEUTRALITY OF

ECCENTRICALLY COATED ELASTIC INCLUSIONS 


\title{
NEUTRALITY OF ECCENTRICALLY COATED ELASTIC INCLUSIONS
}

\author{
Xu Wang And Peter Schiavone
}

In the analysis of neutral coated circular holes in an isotropic medium, it is wellknown that neutrality to a general class of applied uniform fields can be realized only by the concentrically coated circle construction. It is of interest to examine to what degree eccentric circular coatings can be used to achieve effective or near-neutrality in the presence of a wider and more general class of applied fields. To this end, we consider the neutrality of a circular elastic inclusion bonded to its surrounding matrix through $N-2$ eccentric circular coatings $(N \geq 3)$ when the matrix is subjected to remote nonuniform antiplane shear stresses characterized by arbitrary polynomials of order $M \leq N-2$. In our design, the first $N-M-1$ generalized polarization tensors associated with the $N$-phase structure vanish. Our results demonstrate conclusively that for arbitrary applied nonuniform fields, the stress disturbance in the matrix becomes negligible as $N$ becomes sufficiently large, indicating that the inclusion can be made "near-neutral" for a given $N$ and completely neutral as $N$ approaches infinity.

\section{Introduction}

The idea of a "neutral hole" was initiated by Mansfield [1953] who found that certain reinforced holes in a uniformly stressed plate do not alter the original stress field in the uncut body. In other words, the hole shape and corresponding reinforcing layer could be designed to make the hole "invisible" to the surrounding stress field. This idea was later extended to the concept of a "neutral inclusion" in which the insertion of certain shapes of inclusion into an elastic body causes no disturbance in the body's original stress field. In this case, "neutrality" is achieved by adding one or more specifically designed coatings between the inclusion and the surrounding body (see [Milton and Serkov 2001; Chen et al. 2002; Schiavone 2003; Mahboob and Schiavone 2005; Vasudevan and Schiavone 2006; Bertoldi et al. 2007; Benveniste and Miloh 2007; Jarczyk and Mityushev 2012; Wang and

\section{Communicated by Francesco dell'Isola.}

MSC2010: primary 74B05; secondary 74M25.

Keywords: neutral inclusion, remote nonuniform loading, multiple coating, Apollonius circles, generalized polarization tensor. 
Schiavone 2012a; 2012b] for a comprehensive account of fundamental investigations in this area). This concept of "neutrality" finds significant application in the design of advanced composite materials and structures (for example, in the design of implants in biomechanics) but is also topical in that it is often taken to be equivalent to the modern ideas of "cloaking", "invisibility" or "stealth" [Milton et al. 2006; Liu 2010; Ammari et al. 2013a; 2013b] in that the inclusion becomes "invisible" to the original stress distribution.

Milton and Serkov [2001] showed that, for an isotropic medium, neutrality to multiple applied uniform fields can be realized only by the concentrically coated circle construction. This fact has also been observed by $\mathrm{Ru}$ [1998]. In the present paper we intend to show that a circular elastic inclusion with $N-2$ eccentric coatings can be made "almost neutral" to multiple applied nonuniform fields. In fact, we show that when the matrix surrounding the inclusion is subjected to remote nonuniform stresses characterized by arbitrary polynomials of order $M \leq N-2$ in the complex variable $z$, the generalized polarization tensors (GPTs) [Ammari et al. 2013a] of up to order $N-M-1$ vanish on the introduction of the $N-2$ eccentric coatings. For a sufficiently large value of $N$ and a relatively low value of $M$, the disturbance in the matrix is minimal since only GPTs of orders higher than $N-M-1$ exist. As $N$ approaches infinity, there will be no stress disturbance in the matrix as a result of the cancellation of all GPTs.

\section{Design of neutral circular inclusions with multiple eccentric coatings}

Let $\left(x_{1}, x_{2}, x_{3}\right)$ describe a Cartesian coordinate system in $\mathbb{R}^{3}$. In the theory of antiplane shear deformations, the out-of-plane displacement $w\left(x_{1}, x_{2}\right)$, the stress function $\phi\left(x_{1}, x_{2}\right)$, and the stress components $\sigma_{32}\left(x_{1}, x_{2}\right)$ and $\sigma_{31}\left(x_{1}, x_{2}\right)$, can be expressed more conveniently in terms of an analytic function $f(z)$ of the complex variable $z=x_{1}+i x_{2}=r \exp (i \theta)$ where $r=\sqrt{x_{1}^{2}+x_{2}^{2}}$ and $\tan \theta=x_{2} / x_{1}$, as

$$
\mu^{-1} \phi+i w=f(z), \quad \sigma_{32}+i \sigma_{31}=\mu f^{\prime}(z),
$$

where $\mu$ is the shear modulus of the material. The two stress components can be expressed in terms of the stress function $\phi$ as [Ting 1996]

$$
\sigma_{32}=\phi_{, 1}, \quad \sigma_{31}=-\phi_{, 2},
$$

where the notation $(\cdot)_{, s}$ denotes differentiation with respect to $x_{s}, s=1,2$.

We consider a circular elastic inclusion bonded to the surrounding matrix through $N-2$ eccentric circular coatings (Figure 1). Let $S_{1}$ denote the unbounded matrix, $S_{2}, \ldots, S_{N-1}$ the $N-2$ intermediate coatings, and $S_{N}$ the inner circular inclusion. We assume perfect bonding across the $N-1$ eccentric circles $L_{1}, \ldots, L_{N-1}$. Clearly, the interface $L_{k}$ is formed by the outer $S_{k}$ and the inner $S_{k+1}$. The center 


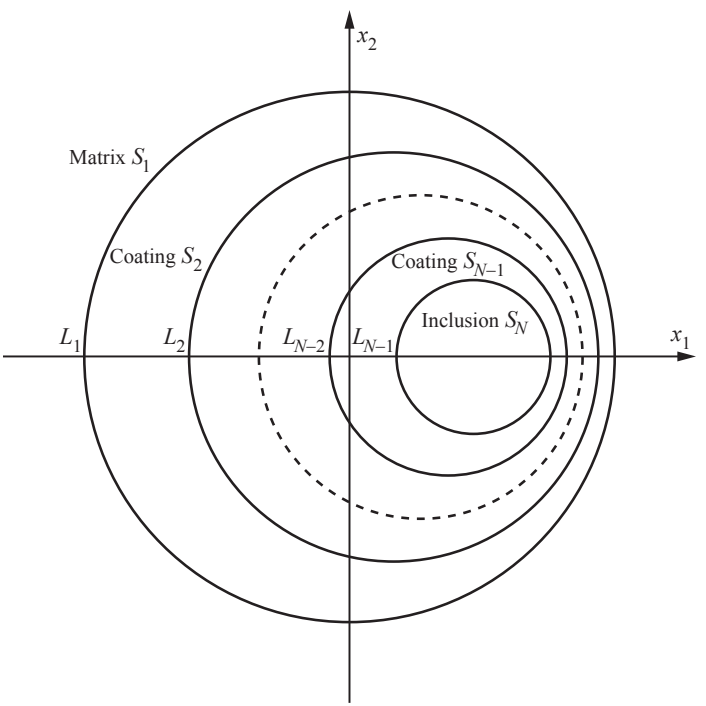

Figure 1. A circular elastic inclusion with $(N-2)$ eccentric circular coatings.

of the unit circle $L_{1}$ is at the origin, so that $L_{1}, \ldots, L_{N-1}$ are Apollonius circles in the sense that, if we introduce the conformal map

$$
z=\omega(\xi)=\frac{\xi-a}{a \xi-1}, \quad \xi=\omega^{-1}(z)=\frac{z-a}{a z-1} \quad(a>1)
$$

then the eccentric circles $L_{1}, \ldots, L_{N-1}$ in the $z$-plane are mapped onto the $N-1$ concentric circles $|\xi|=R_{1}, \ldots,|\xi|=R_{N-1}$ in the $\xi$-plane, respectively, where $R_{N-1}>R_{N-2}>\cdots>R_{2}>R_{1}=1$, as shown in Figure 2. It follows from (3) that (i) the Apollonius circles $L_{k}$ can be described by

$$
\frac{|z-a|}{|a z-1|}=R_{k}
$$

which implies that the centers of these eccentric circles are all on the real axis; (ii) $z=a$ in $S_{1}$ is mapped to $\xi=0$, and $z=1 / a$ in $S_{N}$ is mapped to $\xi=\infty$. In addition, we first assume that the matrix is subjected to remote uniform antiplane stresses $\sigma_{32}^{\infty}$ and $\sigma_{31}^{\infty}$ (it will be seen in the following analysis that the remote applied stresses can be nonuniform). Throughout the remainder of this paper, the subscript $j$ or the superscript $(j)$ will denote the corresponding quantities associated with $S_{j}$. For convenience and without loss of generality, we adopt the notation $f(z)=f(\omega(\xi))=f(\xi)$. 


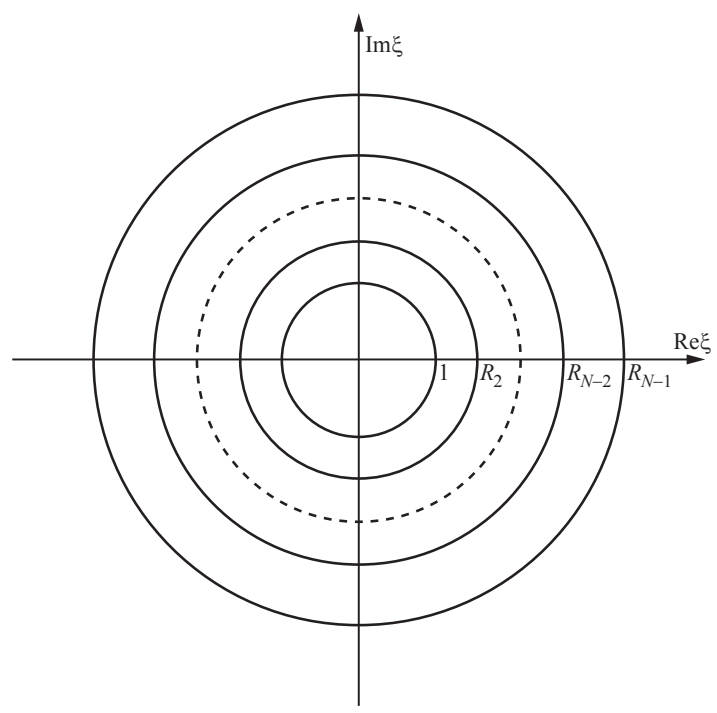

Figure 2. The mapped $\xi$-plane.

The analytic function $f_{j}(\xi)$ defined in phase $j$ can be expanded into the convergent Laurent series

$$
f_{j}(\xi)=\sum_{n=1}^{\infty}\left[A_{n}^{(j)} \xi^{-n}+B_{n}^{(j)} \xi^{n}\right],
$$

where $A_{n}^{(j)}$ and $B_{n}^{(j)}$ are complex constants to be determined (note that we do not include the constant term $(n=0)$ in the Laurent expansion since this term corresponds to a rigid body translation and does not affect the corresponding stress field). It should be pointed out that this expansion of $f_{j}(\xi)$ for $j=1$ is convergent only for $1 / a<|\xi|<1$, and the convergent expression for $f_{1}(\xi)$ in $|\xi|<1$ is

$$
f_{1}(\xi)=\frac{C}{a \xi-1}+\sum_{n=1}^{\infty} B_{n}^{(1)} \xi^{n}, \quad|\xi|<1,
$$

where the complex constant $C$ is determined from the remote uniform stresses as

$$
C=\frac{\left(a^{-1}-a\right)\left(\sigma_{32}^{\infty}+i \sigma_{31}^{\infty}\right)}{\mu_{1}}
$$

Remark 1. Our reasoning in obtaining (5) is as follows. In the physical $z$-plane,

$$
f_{1}(z)=\frac{\sigma_{32}^{\infty}+i \sigma_{31}^{\infty}}{\mu_{1}} z+f_{0}(z), \quad|z|>1,
$$


where $f_{0}(z)$ is analytic everywhere in the matrix, including the point at infinity. Thus, in the mapped $\xi$-plane,

$$
f_{1}(\xi)=\frac{C}{a \xi-1}+f_{0}(\xi), \quad|\xi|<1,
$$

where $f_{0}(\xi)$ is analytic in $|\xi|<1$ and can be expanded in a Taylor series. Consequently, (5) can be obtained with the constant term disregarded.

By enforcing the continuity condition of displacement and traction across the (perfect) interface $|\xi|=R_{j}$ (i.e., $\phi_{j}=\phi_{j+1}, w_{j}=w_{j+1}$ on $|\xi|=R_{j}$ ), we arrive at the recurrence relation

$$
\left[\begin{array}{l}
A_{n}^{(j+1)} \\
\bar{B}_{n}^{(j+1)}
\end{array}\right]=\boldsymbol{P}_{n}^{(j)}\left[\begin{array}{c}
A_{n}^{(j)} \\
\bar{B}_{n}^{(j)}
\end{array}\right], \quad n=1,2, \ldots,
$$

where the transfer matrix $\boldsymbol{P}_{n}^{(j)}$ is given by

$$
\boldsymbol{P}_{n}^{(j)}=\frac{1}{1-\lambda_{j}}\left[\begin{array}{cc}
1 & R_{j}^{2 n} \lambda_{j} \\
R_{j}^{-2 n} \lambda_{j} & 1
\end{array}\right],
$$

with $\lambda_{j}$ being the mismatch parameter defined by

$$
\lambda_{j}=\frac{\mu_{j}-\mu_{j+1}}{\mu_{j}+\mu_{j+1}} \quad\left(\left|\lambda_{j}\right|<1\right) .
$$

It follows from (7) that

$$
\left[\begin{array}{c}
A_{n}^{(N)} \\
\bar{B}_{n}^{(N)}
\end{array}\right]=S_{n}\left[\begin{array}{c}
A_{n}^{(1)} \\
\bar{B}_{n}^{(1)}
\end{array}\right], \quad n=1,2, \ldots,
$$

where

$$
\boldsymbol{S}_{n}=\left[\begin{array}{ll}
S_{n}^{11} & S_{n}^{12} \\
S_{n}^{21} & S_{n}^{22}
\end{array}\right]=\boldsymbol{P}_{n}^{(N-1)} \boldsymbol{P}_{n}^{(N-2)} \cdots \boldsymbol{P}_{n}^{(2)} \boldsymbol{P}_{n}^{(1)}
$$

In order to ensure that $f_{N}(\xi)$ is analytic in the region $R_{N-1}<|\xi|<\infty$, including the point at infinity, we must have $B_{n}^{(N)}=0(n=1,2, \ldots)$. In addition, it can be easily deduced from (4) and (5) that

$$
A_{n}^{(1)}=C a^{-n}, \quad n=1,2, \ldots
$$

By imposing the above additional conditions on (10), we arrive at

$$
B_{n}^{(1)}=-\frac{\bar{C}}{a^{n}} \frac{S_{n}^{21}}{S_{n}^{22}}, \quad n=1,2, \ldots
$$


Thus $f_{1}(\xi)$ defined in the matrix can be uniquely determined as

$$
f_{1}(\xi)=\frac{C}{a \xi-1}-\bar{C} \sum_{n=1}^{\infty}\left(a^{-n} \xi^{n} \frac{S_{n}^{21}}{S_{n}^{22}}\right), \quad(|\xi|<1) .
$$

In order to arrive at a GPT-vanishing structure of order $N-2$, the following $N-2$ conditions should be satisfied:

$$
g^{(k)}\left(a^{-1}\right)=0, \quad k=1,2, \ldots, N-2,
$$

where the superscript $(k)$ denotes the $k$-th order derivative, and

$$
g(\xi)=f_{1}(\xi)-\frac{C}{a \xi-1} .
$$

Remark 2. The conditions given by (15) result in the following asymptotic behavior of $f_{1}(z)$ at infinity:

$$
f_{1}(z) \cong \frac{\sigma_{32}^{\infty}+i \sigma_{31}^{\infty}}{\mu_{1}} z+O\left(1 / z^{N-1}\right) \quad \text { as }|z| \rightarrow \infty,
$$

which indicates that the GPTs up to order $N-2$ all vanish.

In view of (14), (15) can be written explicitly as

$$
\begin{array}{r}
\sum_{n=1}^{\infty}\left[n a^{-2 n} \frac{S_{n}^{21}}{S_{n}^{22}}\right]=0, \\
\sum_{n=1}^{\infty}\left[n(n-1) a^{-2 n} \frac{S_{n}^{21}}{S_{n}^{22}}\right]=0, \\
\vdots \\
\sum_{n=1}^{\infty}\left[n(n-1) \cdots(n+3-N) a^{-2 n} \frac{S_{n}^{21}}{S_{n}^{22}}\right]=0,
\end{array}
$$

which are independent of the remote uniform stresses characterized by the complex constant $C$. In addition, we have the following more general result.

Theorem 1. Equation (17) is also the condition leading to a GPT-vanishing structure of order $N-M-1$ with $M \leq N-2$ when the matrix is subjected to remote nonuniform stresses characterized by

$$
f_{1}(z) \cong \sum_{n=1}^{M} D_{n} z^{n}+O\left(1 / z^{N-M}\right), \quad M \leq N-2, \quad \text { as }|z| \rightarrow \infty,
$$

where $D_{n}(n=1,2, \ldots, M)$ are complex constants. 
Remark 3. In writing (18), it has been implied that the GPTs up to the order $N-M-1$ vanish. As $N$ approaches infinity, keeping $M$ finite, the inclusion will become ideally neutral to arbitrary remote nonuniform stresses.

Proof of Theorem 1. In the region $|\xi|<1, f_{1}(\xi)$ can be written in the convergent form

$$
f_{1}(\xi)=\sum_{n=1}^{M} \frac{C_{n}}{(a \xi-1)^{n}}+\sum_{n=1}^{\infty} B_{n}^{(1)} \xi^{n}, \quad|\xi|<1,
$$

where the complex constants $C_{n}$ can be determined from the nonuniform remote loading characterized by (18).

Through satisfaction of the continuity conditions of traction and displacement across all the existing interfaces, $f_{1}(\xi)$ can be finally determined as

$$
\begin{aligned}
f_{1}(\xi) & =\sum_{n=1}^{M} \frac{C_{n}}{(a \xi-1)^{n}} \\
& -\sum_{n=1}^{\infty}\left[\bar{C}_{1}+\sum_{m=2}^{M} \bar{C}_{m} \frac{(n-1)(n-2) \cdots(n-m+1)}{(m-1) !}\right] \frac{S_{n}^{21}}{S_{n}^{22}} \frac{\xi^{n}}{a^{n}}, \quad|\xi|<1 .
\end{aligned}
$$

If we define the function

$$
h(\xi)=f_{1}(\xi)-\sum_{n=1}^{M} \frac{C_{n}}{(a \xi-1)^{n}},
$$

the $N-2$ conditions in (17) will lead to $h^{(k)}\left(a^{-1}\right)=0,(k=1,2, \ldots, N-M-1)$. This fact implies that the GPTs up to order $N-M-1$ vanish. This completes the proof.

If the $N-1$ geometric parameters $a$ and $R_{2}, R_{3}, \ldots, R_{N-1}$ are given, (17) can be considered as a set of $N-2$ nonlinear equations for the $N-1$ mismatch parameters $\lambda_{1}, \lambda_{2}, \ldots, \lambda_{N-1}$, which can be solved through iteration. In addition, it can be shown that if $\left(\lambda_{1}, \lambda_{2}, \ldots, \lambda_{N-1}\right)$ is a solution to $(17)$, then $\left(-\lambda_{1},-\lambda_{2}, \ldots,-\lambda_{N-1}\right)$ is also a solution.

For example, when $N=4$, (17) becomes

$$
\begin{array}{r}
\sum_{n=1}^{\infty}\left[n a^{-2 n} \frac{\lambda_{1}+R_{2}^{-2 n} \lambda_{2}+R_{3}^{-2 n} \lambda_{3}+R_{2}^{2 n} R_{3}^{-2 n} \lambda_{1} \lambda_{2} \lambda_{3}}{1+R_{2}^{-2 n} \lambda_{1} \lambda_{2}+R_{3}^{-2 n} \lambda_{1} \lambda_{3}+R_{2}^{2 n} R_{3}^{-2 n} \lambda_{2} \lambda_{3}}\right]=0, \\
\sum_{n=1}^{\infty}\left[n(n-1) a^{-2 n} \frac{\lambda_{1}+R_{2}^{-2 n} \lambda_{2}+R_{3}^{-2 n} \lambda_{3}+R_{2}^{2 n} R_{3}^{-2 n} \lambda_{1} \lambda_{2} \lambda_{3}}{1+R_{2}^{-2 n} \lambda_{1} \lambda_{2}+R_{3}^{-2 n} \lambda_{1} \lambda_{3}+R_{2}^{2 n} R_{3}^{-2 n} \lambda_{2} \lambda_{3}}\right]=0 .
\end{array}
$$




\section{Results and discussions}

In this section, we will present numerical results for the cases $N=3, N=4$ and $N \geq 5$. It is of interest to note that very simple approximate closed-form solutions for $N=3$ and $N=4$ can be obtained which, in turn, can be used to quickly (albeit roughly) determine the values of the mismatch parameters. For simplicity it is assumed that the matrix is subjected to only remote uniform stresses $(M=1)$.

3.1. GPT-vanishing structures of order $1(N=3)$. In the case of $N=3$, the following single nonlinear equation should be solved:

$$
\sum_{n=1}^{\infty}\left[n a^{-2 n} \frac{\lambda_{1}+R_{2}^{-2 n} \lambda_{2}}{1+R_{2}^{-2 n} \lambda_{1} \lambda_{2}}\right]=0 .
$$

In view of the fact that $R_{2}>1$ and $\left|\lambda_{1}\right|,\left|\lambda_{2}\right|<1$, the denominator on the left-hand side of (23) can be taken as approximately equal to one (i.e., $1+R_{2}^{-2 n} \lambda_{1} \lambda_{2} \approx 1$ ). Consequently, the following approximate closed-form solution is obtained:

$$
\frac{\lambda_{1}}{\lambda_{2}} \approx-\frac{R_{2}^{2}\left(a^{2}-1\right)^{2}}{\left(a^{2} R_{2}^{2}-1\right)^{2}}=-R_{0}^{2},
$$

where $R_{0}$ is the radius of the inner circular inclusion. We recall that this is just the condition for the existence of a neutral three-phase inclusion with two concentric circular interfaces with radii $R_{0}$ and $1\left(R_{0}<1\right)$ [Ammari et al. 2013a; $\mathrm{Ru} 1999$ ]. This implies that if a concentrically single-coated inclusion is neutral to a remote uniform stress field, the GPT of order 1 of the shifted structure nearly vanishes. We illustrate in Figure 3 the values of $\left(\lambda_{1}, \lambda_{2}\right)$ found for four different values of $a$, namely $a=1.2,1.5,2,10$, with $R_{2}=1.5$. The solid lines are obtained by iteratively solving (23), whilst the dashed lines are obtained by using the approximate solution (24). It is observed that the approximate results are very close to the exact ones. As $a \rightarrow \infty$ (i.e., the eccentricity becomes minimal), (24) simply recovers the exact solution.

3.2. GPT-vanishing structures of order $2(N=4)$. In the case of $N=4$, the equation (22) should be solved iteratively. In addition, the following approximate closed-form solution can be derived:

If $R_{3} \neq R_{2}^{2}$, we have the approximate solution

$$
\begin{aligned}
& \lambda_{2} \approx \frac{-c_{1}+\sqrt{c_{1}^{2}-4 c_{0} c_{2}}}{2 c_{2}}, \\
& \lambda_{1} \approx-\frac{\left(a^{2}-1\right)^{2}\left(a^{2} R_{3}^{2}-R_{2}^{2}\right)^{2}\left[R_{2}^{2}\left(a^{2} R_{3}^{2}-1\right)^{2} \lambda_{2}+R_{3}^{2}\left(a^{2} R_{2}^{2}-1\right)^{2} \lambda_{3}\right]}{\left(a^{2} R_{2}^{2}-1\right)^{2}\left(a^{2} R_{3}^{2}-1\right)^{2}\left[\left(a^{2} R_{3}^{2}-R_{2}^{2}\right)^{2}+R_{2}^{2} R_{3}^{2}\left(a^{2}-1\right)^{2} \lambda_{2} \lambda_{3}\right]},
\end{aligned}
$$




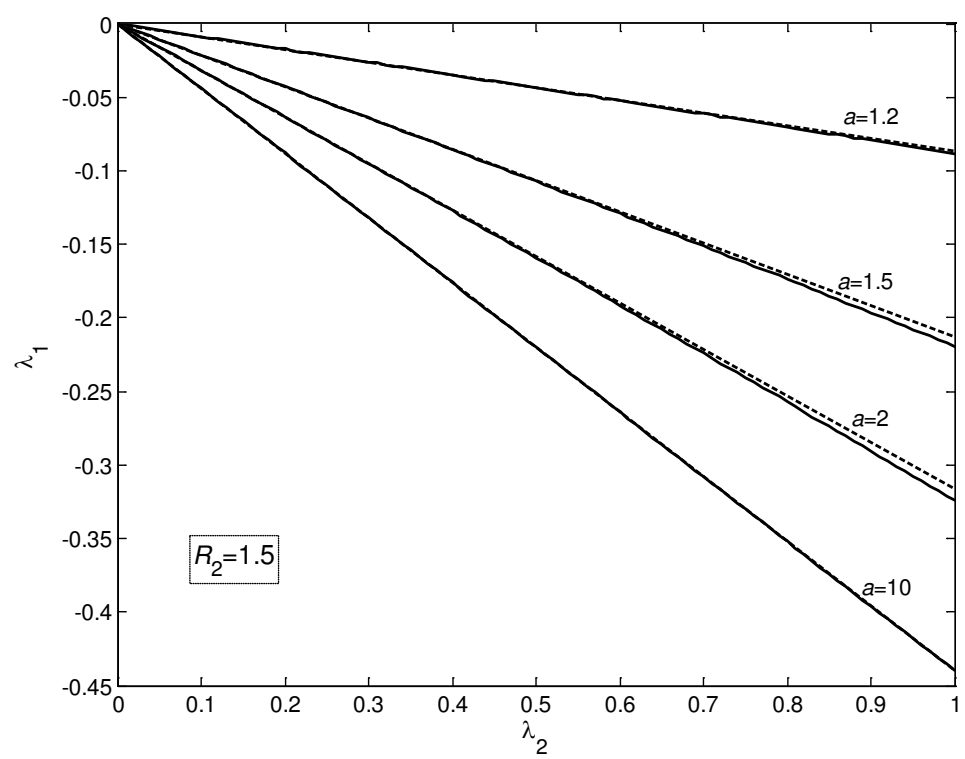

Figure 3. Obtained values of $\left(\lambda_{1}, \lambda_{2}\right)$ for $a=1.2,1.5,2,10$, with $R_{2}=1.5$.

where

$$
\begin{aligned}
& c_{2}=R_{2}^{4} R_{3}^{2}\left(a^{2}-1\right)^{3}\left(a^{2} R_{3}^{2}-1\right)^{3}\left(R_{2}^{4}-R_{3}^{2}\right) \lambda_{3}, \\
& c_{1}=R_{2}^{2}\left(R_{2}^{2}-1\right)\left[R_{3}^{6}\left(a^{2}-1\right)^{3}\left(a^{2} R_{2}^{2}-1\right)^{3} \lambda_{3}^{2}+\left(a^{2} R_{3}^{2}-1\right)^{3}\left(a^{2} R_{3}^{2}-R_{2}^{2}\right)^{3}\right], \\
& c_{0}=R_{3}^{2}\left(R_{3}^{2}-1\right)\left(a^{2} R_{3}^{2}-R_{2}^{2}\right)^{3}\left(a^{2} R_{2}^{2}-1\right)^{3} \lambda_{3} .
\end{aligned}
$$

On the other hand, if $R_{3}=R_{2}^{2}$, we have the approximate solution

$$
\begin{aligned}
& \lambda_{2} \approx- \frac{\lambda_{3} R_{3}\left(R_{3}+1\right)\left(a^{2} R_{3}-1\right)^{3}}{R_{3}^{3}\left(a^{2}-1\right)^{3} \lambda_{3}^{2}+\left(a^{2} R_{3}^{2}-1\right)^{3}}, \\
& \lambda_{1} \approx \frac{\lambda_{3} R_{3}^{3}\left(a^{2}-1\right)^{3}\left[R_{3}\left(a^{2}-1\right)^{3} \lambda_{3}^{2}+\left(a^{2} R_{3}^{2}-1\right)^{3}\right]}{\left(a^{2} R_{3}^{2}-1\right)^{3}\left[R_{3}^{3}\left(a^{2}-1\right)^{3} \lambda_{3}^{2}+\left(a^{2} R_{3}^{2}-1\right)^{3}\right]} \\
& \quad \times \frac{R_{3}^{2}\left(a^{2}-1\right)^{2} \lambda_{3}^{2}-\left(a^{2} R_{3}^{2}-1\right)^{2}}{\left(a^{2}-1\right)^{2} \lambda_{3}^{2}-\left(a^{2} R_{3}^{2}-1\right)^{2}} .
\end{aligned}
$$

We illustrate in Figure 4 the variations of $\lambda_{1}$ and $\lambda_{2}$ as functions of $\lambda_{3}$ for three values of $a$, namely $a=1.01,1.5,3$, with $R_{2}=1.5, R_{3}=2$. The solid lines are the exact results obtained by iteratively solving (22), whereas the dashed lines are the approximate results found from (25). It is observed from Figure 4 that the approximate results are quite satisfactory, especially when $\lambda_{3}<0.7$. 


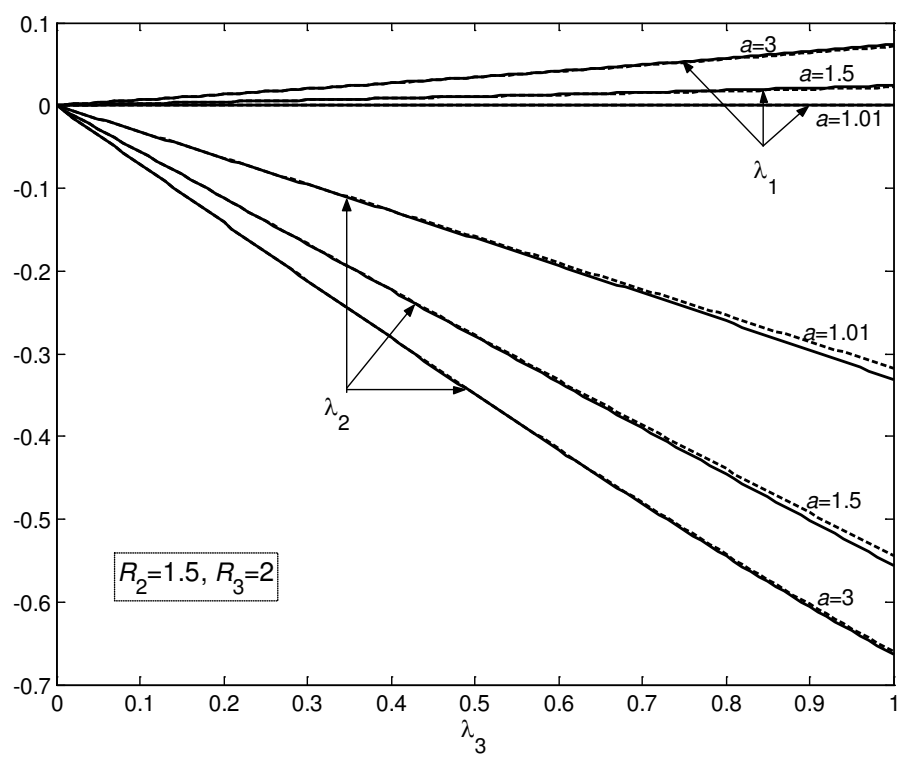

Figure 4. Variations of $\lambda_{1}$ and $\lambda_{2}$ as functions of $\lambda_{3}$ for $a=$ $1.01,1.5,3$, with $R_{2}=1.5, R_{3}=2$.

3.3. GPT-vanishing structures of order 3 or higher $(N \geq 5)$. When $N \geq 5$, the solutions can be obtained only by solving (17) iteratively. Listed in Table 1 are typical results. In performing the calculations, we set the $N-1$ geometric parameters to $a=1.2$ and $R_{k+1}=1+k /(N-2),(k=1,2, \ldots, N-2)$. It is observed that $\lambda_{k}$ and $\lambda_{k+1}$ always have opposite signs.

3.4. Stress disturbance in the matrix. The concept of neutral holes and inclusions was originally proposed to completely eliminate stress concentrations in the matrix [Mansfield 1953; Milton and Serkov 2001; Ru 1998]. In our design, however, the stress disturbance in the matrix cannot be completely avoided due to the existence of GPTs of orders higher than $N-2$ when the remote loading is uniform. However,

\begin{tabular}{rcccccc}
\hline & $N=5$ & $N=6$ & $N=7$ & $N=8$ & $N=9$ & $N=10$ \\
\hline$\lambda_{1}$ & $5.464 \times 10^{-4}$ & $4.203 \times 10^{-5}$ & $4.42 \times 10^{-6}$ & $4.289 \times 10^{-7}$ & $3.613 \times 10^{-8}$ & $2.221 \times 10^{-9}$ \\
$-\lambda_{2}$ & 0.1075 & 0.0147 & 0.0024 & $3.197 \times 10^{-4}$ & $3.573 \times 10^{-5}$ & $2.800 \times 10^{-6}$ \\
$\lambda_{3}$ & 0.6415 & 0.2041 & 0.0592 & 0.0131 & 0.0022 & $2.471 \times 10^{-4}$ \\
$-\lambda_{4}$ & 0.8 & 0.5751 & 0.3239 & 0.1225 & 0.0323 & 0.0053 \\
$\lambda_{5}$ & & 0.5 & 0.5868 & 0.3964 & 0.1720 & 0.0430 \\
$-\lambda_{6}$ & & & 0.4 & 0.5437 & 0.3979 & 0.1594 \\
$\lambda_{7}$ & & & & 0.3 & 0.4445 & 0.2939 \\
$-\lambda_{8}$ & & & & & 0.2 & 0.2728 \\
$\lambda_{9}$ & & & & & & 0.1 \\
\hline
\end{tabular}

Table 1. Obtained mismatch parameters for $N=5,6,7,8,9,10$. 


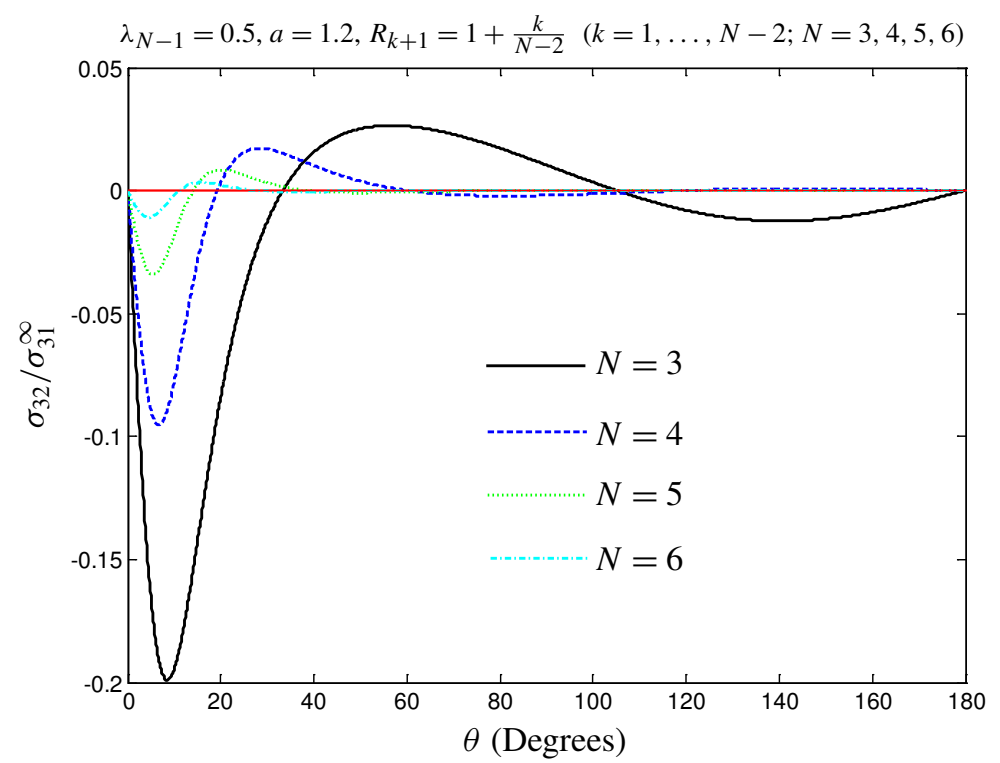

Figure 5. The stress disturbance $\sigma_{32} / \sigma_{31}^{\infty}$ along the coating/matrix interface $|z|=1$ on the matrix side for $N=3,4,5,6$ when the matrix is subjected to $\sigma_{31} \neq 0, \sigma_{32}^{\infty}=0$.

the stress disturbance is expected to be insignificant as $N$ becomes sufficiently large. In fact, we illustrate in Figures 5 and 6 the stress disturbance along the coating/matrix interface $|z|=1$ on the matrix side for $N=3,4,5,6$ when the matrix is subjected to the loading given by $\sigma_{31}^{\infty} \neq 0, \sigma_{32}^{\infty}=0$. It is observed from the two figures that, as $N$ increases, the most significant stress disturbance occurs in a more localized region of $\theta: \theta<60^{\circ}, 40^{\circ}$ and $20^{\circ}$ for $N=4,5$ and 6 , respectively. Clearly, when $N=6$, the stress disturbance along the whole interface $|z|=1$ is minimal.

\section{Conclusions}

By adopting the GPT cancellation method proposed in [Ammari et al. 2013a], we design "near-neutral" circular elastic inclusions with multiple eccentric circular coatings. When the matrix is subjected to remote nonuniform stress characterized by (18), the GPTs up to the $(N-M-1)$-th order are canceled by appropriately adding $N-2$ eccentric coatings between the inclusion and the matrix. Condition (17) is, in fact, independent of the remote applied nonuniform loading given by (18). Consequently, our design of an $N$-phase circular inclusion is "almost neutral" to the remote nonuniform stresses characterized by any polynomials in $z$ of order $M$ no greater than $N-2$. In order to make the analysis tractable, we 


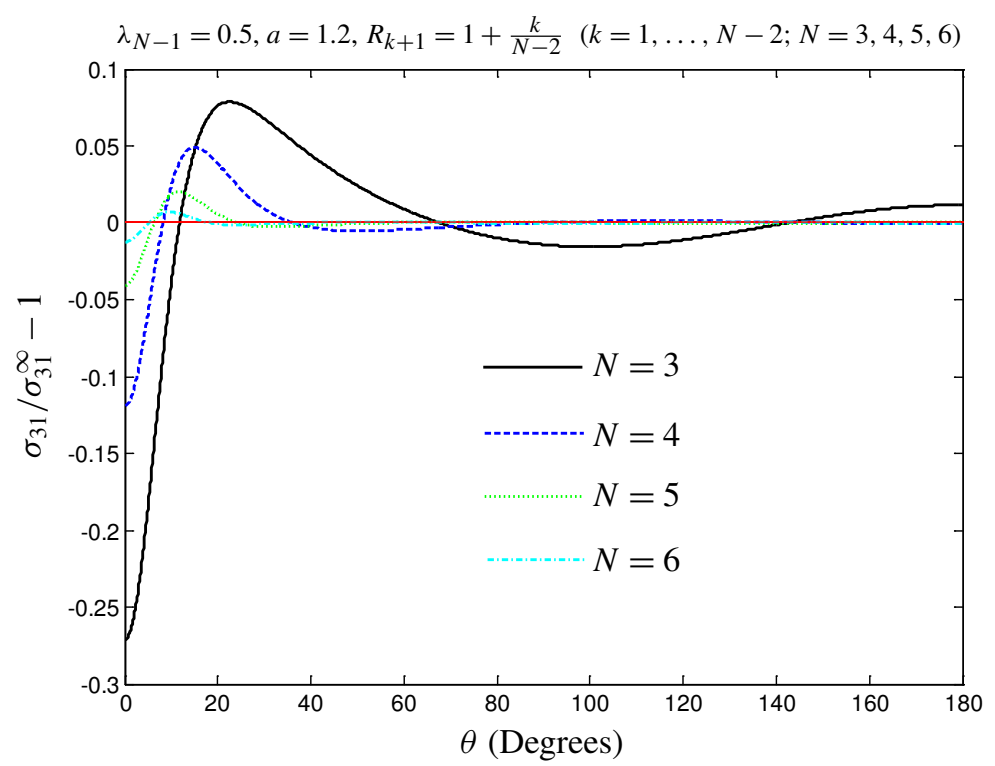

Figure 6. The stress disturbance $\sigma_{31} / \sigma_{31}^{\infty}-1$ along the coating/matrix interface $|z|=1$ on the matrix side for $N=3,4,5,6$ when the matrix is subjected to $\sigma_{31}^{\infty} \neq 0, \sigma_{32}^{\infty}=0$.

assume that all the eccentric circular interfaces $L_{k}(k=1,2, \ldots, N-1)$ are Apollonius circles. Approximate closed-form solutions (24) for $N=3$ and (25)-(27) for $N=4$ are obtained. One consequence and potential application of the results here arises from the finding that multiple closely spaced and eccentrically coated inclusions can be inserted into a nonuniformly stressed finite matrix with minimal stress disturbance in the matrix.

\section{Acknowledgements}

The authors are indebted to two very meticulous reviewers, whose comments greatly improved the paper. This work is supported by the National Natural Science Foundation of China (grant number 11272121) and through a Discovery Grant from the Natural Sciences and Engineering Research Council of Canada.

\section{References}

[Ammari et al. 2013a] H. Ammari, H. Kang, H. Lee, and M. Lim, "Enhancement of near cloaking using generalized polarization tensors vanishing structures, I: The conductivity problem", Comm. Math. Phys. 317:1 (2013), 253-266. arXiv 1104.3936v1

[Ammari et al. 2013b] H. Ammari, H. Kang, H. Lee, and M. Lim, "Enhancement of near-cloaking, II: The Helmholtz equation”, Comm. Math. Phys. 317:2 (2013), 485-502. arXiv 1110.1922v1 
[Benveniste and Miloh 2007] Y. Benveniste and T. Miloh, "Soft neutral elastic inhomogeneities with membrane-type interface conditions", J. Elasticity 88:2 (2007), 87-111.

[Bertoldi et al. 2007] K. Bertoldi, D. Bigoni, and W. J. Drugan, "Structural interfaces in linear elasticity, II: Effective properties and neutrality”, J. Mech. Phys. Solids 55:1 (2007), 35-63.

[Chen et al. 2002] T. Chen, Y. Benveniste, and P. C. Chuang, "Exact solutions in torsion of composite bars: thickly coated neutral inhomogeneities and composite cylinder assemblages", Proc. R. Soc. Lond. A 458:2023 (2002), 1719-1759.

[Jarczyk and Mityushev 2012] P. Jarczyk and V. Mityushev, "Neutral coated inclusions of finite conductivity”, Proc. R. Soc. Lond. A 468:2140 (2012), 954-970.

[Liu 2010] L. P. Liu, "Neutral shells and their applications in the design of electromagnetic shields", Proc. R. Soc. Lond. A 466:2124 (2010), 3659-3677.

[Mahboob and Schiavone 2005] M. Mahboob and P. Schiavone, "Designing a neutral elliptic inhomogeneity in the case of a general non-uniform loading", Appl. Math. Lett. 18:11 (2005), 13121318 .

[Mansfield 1953] E. H. Mansfield, "Neutral holes in plane sheet: reinforced holes which are elastically equivalent to the uncut sheet", Quart. J. Mech. Appl. Math. 6 (1953), 370-378.

[Milton and Serkov 2001] G. W. Milton and S. K. Serkov, "Neutral coated inclusions in conductivity and anti-plane elasticity”, Proc. R. Soc. Lond. A 457:2012 (2001), 1973-1997.

[Milton et al. 2006] G. W. Milton, M. Briane, and J. R. Willis, "On cloaking for elasticity and physical equations with a transformation invariant form”, New J. Phys. 8:10 (2006), Article ID \#248.

[Ru 1998] C.-Q. Ru, "Interface design of neutral elastic inclusions”, Int. J. Solids Struct. 35:7-8 (1998), 559-572.

[Ru 1999] C.-Q. Ru, "A new method for an inhomogeneity with stepwise graded interphase under thermomechanical loadings", J. Elasticity 56:2 (1999), 107-127.

[Schiavone 2003] P. Schiavone, "Neutrality of the elliptic inhomogeneity in the case of non-uniform loading”, Int. J. Eng. Sci. 41:18 (2003), 2081-2090.

[Ting 1996] T. C. T. Ting, Anisotropic elasticity: theory and applications, Oxford Engineering Science Series 45, Oxford University Press, New York, 1996.

[Vasudevan and Schiavone 2006] M. Vasudevan and P. Schiavone, "New results concerning the identification of neutral inhomogeneities in plane elasticity", Arch. Mech. Stos. 58:1 (2006), 45-58.

[Wang and Schiavone 2012a] X. Wang and P. Schiavone, "Neutral coated circular inclusions in finite plane elasticity of harmonic materials", Eur. J. Mech. A Solids 33 (2012), 75-81.

[Wang and Schiavone 2012b] X. Wang and P. Schiavone, "Neutrality in the case of $N$-phase elliptical inclusions with internal uniform hydrostatic stresses", Int. J. Solids Struct. 49:5 (2012), 800-807.

Received 1 Nov 2013. Revised 13 Feb 2014. Accepted 21 Apr 2014.

XU WANG: xuwang@ecust.edu.cn

School of Mechanical and Power Engineering, East China University of Science and Technology, 130 Meilong Road, Shanghai, 200237, China

PETER SCHIAVONE: p.schiavone@ualberta.ca

Department of Mechanical Engineering, University of Alberta, 4-9 Mechanical Engineering

Building, Edmonton, Alberta, Canada T6G 2G8 